\title{
Lifestyle of the Elderly with Stroke: A Cross Sectional Study
}

\author{
Somaye Pouy ${ }^{1}$, Masoumeh Otaghi ${ }^{2}$, Milad Borji $^{2}$, Asma Tarjoman $^{3}$ and Parisa Sanei ${ }^{3,{ }^{*}}$ \\ ${ }^{1}$ Guilan University of Medical Sciences, Rasht, Iran \\ ${ }^{2}$ Department of Nursing, Faculty of Nursing and Midwifery, Ilam University of Medical Sciences, Ilam, Iran \\ ${ }^{3}$ Student Research Committee, Ilam University of Medical Sciences, Ilam, Iran \\ "Corresponding author: Student Research Committee, Ilam University of Medical Sciences, Ilam, Iran. Email: sanei.parisa73@gmail.com
}

Received 2018 February 26; Revised 2018 July 06; Accepted 2018 July 11.

\begin{abstract}
Background: Nowadays, stroke is one of the most important and commonly diagnosed neurological diseases throughout the world. This disease annually causes numerous moralities and disabilities; its high prevalence is reported among the elderly. Objectives: The current study aimed at determining the lifestyle of the elderly with stroke in Ilam, Western Iran.

Methods: The current descriptive, cross sectional study was conducted on 200 elderly people with stroke in Ilam, Western Iran by convenience sampling method. The study was conducted by the application of healthy lifestyle questionnaire with confirmed validity and reliability. After data collection, the data were analyzed with SPSS version 16 using descriptive statistics (mean and standard deviation) and inferential statistics (independent $t$ test and ANOVA).

Results: Findings of the current study showed that the majority of the studied elderly were male ( $n=106,53 \%)$ aged 65 - 70 years ( $n$ $=93,46.5 \%)$, educated up to middle school level $(n=112,56 \%)$, with annual income range of $\$ U S 72,000$ to $\$ U S 144,000(n=117,58.5 \%)$, had no spouse $(n=111,5.55 \%)$ and lived in the city $(n=115,57.5 \%)$. Mean ( \pm standard deviation; SD $)$ of age was $75.56 \pm 9.87$ years, mean lifestyle score was $37.54 \pm 3.91,9.55 \pm 1.79,24.30 \pm 3.36,18.76 \pm 1.79$, and $28.04 \pm 2.41$ in the dimensions of prevention, physical activity and recreational activities and sport, healthy nutrition, stress management, and inter-personal relationships, respectively. Total score of the questionnaire was $118.20 \pm 6.00$, which indicated a medium score of healthy lifestyle in the elderly.

Conclusions: Findings of the current study revealed that the lifestyle condition of the elderly with stroke was in medium level. It was suggested that the therapeutic and health teams, in particular nurses, intervene properly and use nursing care models to enhance the lifestyle of the elderly.
\end{abstract}

Keywords: Lifestyle, Elderly, Stroke

\section{Background}

Cerebrovascular diseases are one of the most important and common neurological diseases in the world, including ischemic and hemorrhagic strokes and cerebrovascular anomalies (1). Today, stroke is considered as one of the most common neurological diseases of the world, which annually causes a great number of deaths and disabilities $(2,3)$. In this regard, the spread of strokeinduced disabilities is one of the challenges of the current century (4). Stroke prevalence among the older adults was reported $46-73$ per 1000 people (5). This rate increased due to prevalence of aging $(6,7)$.

Stroke is the most common disabling neurological disease among the adults, which could result in damages to perception system, sensory, vision, and motor and cognitive abilities; therefore, the ability of patients in performing their daily activities is reduced (8). Stroke may cause complication in the patient such as the increase of risk of falling $(9,10)$, motionlessness or reduction of physical activities (11), decrease of cognitive and social activity (12), and psychological health of the patient (13). Therefore, its prevention is highly essential (14). Hypertension, hyperlipidemia, diabetes, smoking, and obesity are among the factors of stroke. All these factors could be changed by lifestyle modification. Therefore, identification of lifestyle condition of these patients is essential (15).

Results of a study on 40,102 individuals with first-ever ischemic stroke in Denmark showed that the prevalence of some cardiovascular risk factors such as hypertension, diabetes mellitus, coronary heart disease, and, in males, also atrial fibrillation went down after the age 70 to 80 years (16). However, the study by Tanimura et al. (17), indicated that self-care agency, lifestyle, and physical condition could predict future frailty in the elderly and they suggested that enhancing self-care agency, regular exercise, and self-management skills for chronic illness and disability may decrease the progression of frailty in older adults. 
A leisure world cohort study of 13,978 individuals indicated that not smoking, moderate alcohol consumption, physical activity, and normal weight were important health promoters (18).

Healthy lifestyle provides, protects and enhances the health and welfare of people (19). Indeed, lifestyle is a combination of behavioral patterns and personal habits throughout the person's life including nutrition, physical activity, stress, sleep quality, and smoking, which are formed following the socialization and can affect the health of people (20). Lifestyle last throughout the time and based on its nature, it can form risks or security for the person. This makes the role of lifestyle very effective (21). On the other hand, healthy lifestyle can significantly prevent stroke (22). That is why to develop a healthy community, the healthy lifestyle should be promoted among its members; therefore, the health-related objectives such as encouragement of healthy behaviors and providing healthy social and physical environment should be achieved (23). As aging increases throughout the world (24-26), resolving the needs and challenges of the elderly is one of the social necessities (27). This highlights the necessity of paying attention to the elderly with stroke.

\section{Objectives}

The current study aimed at determining the lifestyle condition of the elderly with stroke in Ilam, Western Iran.

\section{Methods}

\subsection{Study Design}

The current descriptive, cross sectional study was conducted from January to December in 2017 at Shahid Mostafa Khomeini hospital, which is the only hospital to manage, cure, and care the patients with stroke in Ilam. The current study protocol was confirmed by ethical committee of Ilam University of Medical Sciences.

\subsection{Study Population}

Study population included the elderly with stroke referring to Shahid Mostafa Khomeini hospital in Ilam. Diagnosis of stroke was confirmed by the physicians based on the international classification of diseases-10th edition (ICD-10). Inclusion criteria were stroke (based on physician diagnosis in the medical record of the patient), minimum age of 65 years, ability to communicate, living in Ilam, and providing signed informed consent. Exclusion criteria were incomplete questionnaires and unwillingness to participate in the study. Convenience sampling was continued until sample size was completed. Sample size using the below formula was set to 188 with $10 \%$ (18 individuals) dropouts. Totally, 200 subjects completed the study.

$$
\begin{aligned}
n= & \frac{z_{\left(1-\frac{\alpha}{2}\right)}^{2} P(1-P)}{d^{2}} \\
& \alpha=0.05, \mathrm{z}=1.96, \mathrm{P}=0.04, \mathrm{~d}=0.07
\end{aligned}
$$

\subsection{Data Gathering}

The data collection tools included demographic and elderly lifestyle questionnaires; the validity and reliability of the latter questionnaire were determined in a study by Eshaghi et al. (28). The elderly lifestyle questionnaire includes 46 major questions regarding prevention (15 items, score range 15 to 73 ), physical and recreational activities and sport (five items, score range 3 to 17), healthy food (14 items, score range 13 to 55), stress management (five items, score range 5 to 25), and interpersonal and social relationships (seven items, score range 6 to 35). Responsiveness scale of this questionnaire includes the repetition of action and multi-choice scales (single-choice or multi-choice).The option indicating the most undesirable level of lifestyle is assigned with score 1 , the rest of options are assigned with scores 2, 3, 4, and 5. Total scores range 42 to 205 (29).

After getting the permission form the authorities, the researchers referred to Shahid Mostafa Khomeini hospital in the morning and evening shifts on all days of the week including holidays. After getting the recommendations and introducing themselves to research units, the patients with stroke were identified and sampling was started. Then, the objectives of the study were explained to the patients and they were also aware of their ability to withdraw from the study at any time and their information would remain confidential. If the patients were able to fill the questionnaire, they did it by themselves; otherwise, it was completed in an interview. Moreover, if the patients could not answer the questions, they were called in a proper time and were invited to come to the hospital for filling the questionnaires, if they were interested.

\subsection{Data Analysis}

Data were analyzed with SPSS version 16 and by descriptive statistics (mean, SD) and inferential statistics (independent $t$ test and ANOVA).

\section{Results}

According to the findings listed in Table 1 . The majority of the studied elderly were male $(n=106,53 \%)$, aged 65 70 years $(n=93,46.5 \%)$, educated up to middle school level $(\mathrm{n}=112,56 \%$ ), with annual income range of \$US 72,000 to \$US144,000 $(\mathrm{n}=117,58.5 \%)$, had no spouse $(\mathrm{n}=111,5.55 \%)$ and 


\begin{tabular}{|c|c|c|}
\hline Sub-Category & $\mathbf{N}$ & $\%$ \\
\hline \multicolumn{3}{|l|}{ Gender } \\
\hline Male & 106 & 53 \\
\hline Female & 94 & 47 \\
\hline \multicolumn{3}{|l|}{ Age, $y$} \\
\hline $70-65$ & 93 & 46.5 \\
\hline $75-71$ & 23 & 11.5 \\
\hline $80-76$ & 24 & 12 \\
\hline $85-81$ & 33 & 16.5 \\
\hline Above 86 & 27 & 13.5 \\
\hline \multicolumn{3}{|l|}{ Educational level } \\
\hline Elementary & 43 & 21.5 \\
\hline Tips & 112 & 56 \\
\hline High school & 39 & 19.5 \\
\hline Academic & 6 & 3 \\
\hline \multicolumn{3}{|l|}{ Income level, SUS } \\
\hline Less than 72,000 & 34 & 17 \\
\hline $72,000-144,000$ & 117 & 58.5 \\
\hline More than 144,000 & 49 & 24.5 \\
\hline \multicolumn{3}{|l|}{ Marital status } \\
\hline Married & 89 & 44.5 \\
\hline Single & 111 & 55.5 \\
\hline \multicolumn{3}{|l|}{ Residence } \\
\hline City & 115 & 57.5 \\
\hline Village & 85 & 42.5 \\
\hline
\end{tabular}

lived in the city $(\mathrm{n}=115,57.5 \%)$. Mean \pm SD age of the participants was $75.56 \pm 9.87$ years.

Based on Table 2, a significant relationship was observed between the gender, educational level, marital status, and income level, and the lifestyle of the elderly ( $\mathrm{P}<$ 0.05). However, no significant relationship was observed between the age and place of residence, and the lifestyle condition of the patients $(\mathrm{P}>0.05)$. Statistical findings of the current study indicated that the mean $( \pm S D)$ score of lifestyle of the elderly with stroke was $37.54 \pm 3.91$ in the prevention dimention; in terms of physical activity, recreational activities and sport, the score was $9.55 \pm 1.79$. The mean ( \pm SD) score of the elderly lifestyle was $24.30 \pm 3.36$ and $18.76 \pm 1.79$ for healthy diet, and social and interpersonal relationships, respectively. Overall, the mean $( \pm S D)$ score of the study subjects was $118.20 \pm 6.00$ indicating a medium lifestyle.

\section{Discussion}

The current study aimed at determining the lifestyle of the elderly with stroke. Findings revealed that their lifestyle condition was at medium level. The findings of a study by Fallah Mehrabadi et al. (20), on a group of the elderly with osteoporosis referred to bone density center of Tehran University of Medical Sciences Tehran, Iran, revealed that the lifestyle of the subjects was in moderate conditions. Also, Heshmati et al. (30), investigated an elderly group in Kashmar, Iran, and indicated that most of the elderly had a moderate lifestyle, which was consistent with the results of the current study. However, based on the study by Lori et al. (29), on an elderly group in Shiraz, Iran, most of the subjects had a good lifestyle, which was not in line with the results of the current study.

Regarding the current study findings, there was a statistically significant relationship between lifestyle and gender. Moreover, a significant difference was observed between males and females in different dimensions of lifestyle, except for the social and interpersonal relationships. Females' scores were higher than those of males. Babak et al. (19), studied an elderly group in Isfahan, Iran based on different dimension of lifestyle, and reported that males attained significantly higher scores in stress management, physical and recreational activities and sports compared with females. Their findings were inconsistent with those of the current study, although some similarities were found in prevention dimension; however, females' score were higher than that of males. Findings of the study by Najimi et al. (31), on the elderly group living in nursing homes in Isfahan showed no relationship between gender and lifestyle. The difference between the results of the current study and the mentioned studies can be attributed to the fact that the current study was conducted on the elderly with stroke, while other studies enrolled the elderly living in the community (19) or nursing homes (31).

The current study showed no significant relationship between age and the lifestyle domains, which was compatible with the results of the study by Fallah Mehrabadi et al. (20), on the elderly with osteoporosis referred to bone density center of Tehran University of Medical Sciences, and findings of Motaghi and Davari Dolatabadi (26), addressing the elderly group in Isfahan. Motaghi and Davari Dolatabadi (26), indicated no significant relationship between age and lifestyle. Furthermore, in the study by Mahmudi et al. (32), on the aged population of AghQala, Iran, a significant relationship was observed between age, and prevention, sport and recreation, stress, and social relationships that was not in line with the results of the current study. Such inconsistencies can be attributed to different times and locations in the studies. Moreover, it seems that the study by Mahmudi et al. (32), did not target el- 


\begin{tabular}{|c|c|c|c|c|c|c|}
\hline Sub-Categories & Prevention & $\begin{array}{c}\text { Physical and } \\
\text { Recreational Activities } \\
\text { and Sport }\end{array}$ & Healthy Diet & Stress Management & $\begin{array}{l}\text { Interpersonal and Social } \\
\text { Relationships }\end{array}$ & Total \\
\hline \multicolumn{7}{|l|}{ Gender } \\
\hline Male & $35.73 \pm 3.03$ & $9.28 \pm 1.59$ & $22.75 \pm 2.92$ & $18.41 \pm 1.80$ & $27.95 \pm 2.56$ & $114.14 \pm 3.68$ \\
\hline P-value & 0.000 & 0.02 & 0.000 & 0.004 & 0.56 & 0.000 \\
\hline \multicolumn{7}{|l|}{ Age, $y$} \\
\hline $65-70$ & $37.66 \pm 4.29$ & $9.28 \pm 1.55$ & $24.27 \pm 3.35$ & $18.89 \pm 1.69$ & $28.02 \pm 2.71$ & $118.14 \pm 6.83$ \\
\hline $71-75$ & $36.86 \pm 4.94$ & $9.30 \pm 1.79$ & $23.60 \pm 3.36$ & $18.56 \pm 1.99$ & $27.26 \pm 2.19$ & $115.60 \pm 5.51$ \\
\hline $76-80$ & $37.37 \pm 3.42$ & $10.08 \pm 2.32$ & $23.62 \pm 3.28$ & $18.70 \pm 1.65$ & $28.75 \pm 1.84$ & $118.54 \pm 5.59$ \\
\hline $81-86$ & $38.18 \pm 3.19$ & $9.66 \pm 2.11$ & $25.12 \pm 3.03$ & $18.96 \pm 1.96$ & $28.00 \pm 2.13$ & $119.93 \pm 4.56$ \\
\hline$>86$ & $36.88 \pm 3.14$ & $10.03 \pm 1.60$ & $24.44 \pm 3.77$ & $18.55 \pm 1.78$ & $28.44 \pm 2.24$ & $118.37 \pm 5.10$ \\
\hline P-value & 0.66 & 0.17 & 0.41 & 0.82 & 0.27 & 0.12 \\
\hline \multicolumn{7}{|l|}{ Education level } \\
\hline Elementary & $36.53 \pm 3.49$ & $9.25 \pm 1.81$ & $23.44 \pm 3.59$ & $18.81 \pm 1.74$ & $28.81 \pm 2.63$ & $116.86 \pm 3.52$ \\
\hline Tips & $36.43 \pm 3.21$ & $9.33 \pm 1.62$ & $23.83 \pm 2.98$ & $18.50 \pm 1.80$ & $27.74 \pm 2.49$ & $115.83 \pm 4.78$ \\
\hline High school & $40.76 \pm 3.62$ & $10.61 \pm 2.00$ & $25.92 \pm 3.42$ & $19.28 \pm 1.74$ & $28.00 \pm 1.87$ & $124.58 \pm 4.74$ \\
\hline Academics & $44.33 \pm 2.16$ & $9.00 \pm 0.89$ & $28.66 \pm 1.21$ & $19.83 \pm 1.16$ & $28.50 \pm 1.04$ & $130.33 \pm 3.77$ \\
\hline P-value & 0.000 & 0.001 & 0.000 & 0.04 & 0.09 & 0.000 \\
\hline \multicolumn{7}{|l|}{ Income level, SUS } \\
\hline Less than 72,000 & $39.67 \pm 4.70$ & $9.47 \pm 1.63$ & $26.20 \pm 3.15$ & $18.38 \pm 1.74$ & $27.82 \pm 2.65$ & $121.55 \pm 6.33$ \\
\hline More than 144,000 & $35.81 \pm 3.32$ & $9.59 \pm 1.97$ & $22.97 \pm 2.79$ & $18.81 \pm 1.95$ & $28.04 \pm 2.33$ & $115.24 \pm 5.26$ \\
\hline P-value & 0.000 & 0.95 & 0.000 & 0.40 & 0.83 & 0.000 \\
\hline \multicolumn{7}{|l|}{ Marital status } \\
\hline Living with spouse & $38.84 \pm 4.07$ & $9.64 \pm 1.72$ & $25.53 \pm 3.19$ & $19.08 \pm 1.86$ & $28.33 \pm 1.98$ & $121.44 \pm 5.85$ \\
\hline Single & $36.49 \pm 3.45$ & $9.48 \pm 1.86$ & $23.30 \pm 3.18$ & $18.49 \pm 1.69$ & $27.81 \pm 2.68$ & $115.59 \pm 4.72$ \\
\hline P-value & 0.000 & 0.54 & 0.000 & 0.01 & 0.12 & 0.000 \\
\hline \multicolumn{7}{|l|}{ Place of residence } \\
\hline City & $37.54 \pm 3.66$ & $9.79 \pm 1.95$ & $24.17 \pm 3.45$ & $18.81 \pm 1.75$ & $28.12 \pm 2.37$ & $118.45 \pm 6.15$ \\
\hline Village & $37.52 \pm 4.24$ & $9.23 \pm 1.50$ & $24.47 \pm 3.26$ & $18.68 \pm 1.85$ & $27.94 \pm 2.46$ & $117.85 \pm 5.79$ \\
\hline P-value & 0.97 & 0.03 & 0.54 & 0.59 & 0.60 & 0.49 \\
\hline
\end{tabular}

${ }^{a}$ Values are available mean \pm SD.

derly people with a certain disease; while the current study investigated elderly patients with stroke and in the study by Fallah Mehrabadi et al. (20), patients with osteoporosis were examined. These factors all may affect the lifestyle.

Findings of the current study indicated a significant relationship between the lifestyle and education level, and by increase of education level, the lifestyle improved as well. Various other studies also showed improvement of lifestyle by increase of education level $(20,32-34)$, which were in agreement with the current study results. Also, based on the current study, a significant relationship was observed between the marital status and lifestyle, and the married elderly had a better lifestyle. This finding was also in line with numerous other studies in this field $(20,29,31)$.

Findings of the current study indicated that the lifestyle of the elderly with stroke was at medium level. It is suggested that the therapeutic and health teams, in particular nurses, intervene properly and use nursing care prac- 
tices to enhance the lifestyle of the elderly with stroke.

\section{Acknowledgments}

The study was derived from the result of a research project issued by Ilam University of Medical Sciences (IUMS). Authors wish to thank the elderly participants and IUMS to cooperation with the study.

\section{References}

1. Faraji F, Talaei ZA, Eshrati B, Pirasteh S. The evaluation of correlation between plasma level of CRP and WBC with ischemic stroke severity and infarct volume. ARAK MED UJ (AMUJ). 2009;11(4):105-12.

2. Asgari MR, Dehvan F, Ghorbani R, Samaei A, Binesh M, Rahaei F, et al. [Upper extremity motor function and hand muscular power in patients with stroke: A pilot study]. Koomesh. 2017;19(2):438-47. Persian.

3. Jouzi M. [Assessment of the effect of massage therapy on stroke patients]. Med Sci J -Islamic Azad Univesity Tehran Medical Branch. 2009;19(4):256-61. Persian.

4. Hatamabadi HR, Mansourifar H, Asarzadegan F, Shojaee M. [Barriers to On Time Delivery of Thrombolytic Therapy for Patients with Acute Stroke]. J Mazandaran U Med Sci (JMUMS). 2013;23(102). Persian.

5. Feigin VL, Lawes CMM, Bennett DA, Anderson CS. Stroke epidemiology: a review of population-based studies of incidence, prevalence, and case-fatality in the late 20th century. Lancet Neurol. 2003;2(1):4353. doi: 10.1016/s1474-4422(03)00266-7.

6. El Hachioui H, Visch-Brink EG, de Lau LM, van de Sandt-Koenderman MW, Nouwens F, Koudstaal PJ, et al. Screening tests for aphasia in patients with stroke: a systematic review. J Neurol. 2017;264(2):211-20. doi: 10.1007/s00415-016-8170-8. [PubMed: 27260296]. [PubMed Central: PMC5306063].

7. Sottini C, Vangi D, Rizzi E, Stampatori C, Mattioli F. Early Post Stroke Rehabilitation Load Correlates With Greater Functional Improvement of Hemiparesis. Archives of Neuroscience. 2016;4(1). doi: 10.5812/archneurosci.39089.

8. Bahrami M, Mazloom SR, Hasanzadeh F, Ghandehari K. [The Effect of Mirror Therapy on Self-Care stroke patients].J Mazandaran U Med Sci (JMUMS). 2013;23(107). Persian.

9. Xu T, O'Loughlin K, Clemson L, Lannin NA, Dean C, Koh G. Developing a falls prevention program for community-dwelling stroke survivors in Singapore: client and caregiver perspectives. Disabil Rehabil.2017:111. doi: 10.1080/09638288.2017.1419293. [PubMed: 29277114].

10. Azadeh H, Fekri A, Amraie H, Roostaei M, Baharlouei H. The Correlation Between Rates of Falling, Balance, Quality of Life and Fear of Falling in Patients With Chronic Stroke.J Rehabil.2018;19(1):36-43. doi: 10.21859/jrehab.19.1.36.

11. English C, Manns PJ, Tucak C, Bernhardt J. Physical activity and sedentary behaviors in people with stroke living in the community: a systematic review. Phys Ther. 2014;94(2):185-96. doi: 10.2522/ptj.20130175. [PubMed: 24029302].

12. Janssen H, Ada L, Bernhardt J, McElduff P, Pollack M, Nilsson M, et al. Physical, cognitive and social activity levels of stroke patients undergoing rehabilitation within a mixed rehabilitation unit. Clin Rehabil. 2014;28(1):91-101. doi: 10.1177/0269215512466252. [PubMed: 23193176].

13. Crichton SL, Bray BD, McKevitt C, Rudd AG, Wolfe CD. Patient outcomes up to 15 years after stroke: survival, disability, quality of life, cognition and mental health. J Neurol Neurosurg Psychiatry. 2016;87(10):1091-8. doi: 10.1136/jnnp-2016-313361. [PubMed: 27451353].

14. Furie KL, Kasner SE, Adams RJ, Albers GW, Bush RL, Fagan SC, et al. Guidelines for the prevention of stroke in patients with stroke or transient ischemic attack: a guideline for healthcare professionals from the american heart association/american stroke association.
Stroke. 2011;42(1):227-76. doi: 10.1161/STR.0b013e3181f7d043. [PubMed: 20966421].

15. Haji Hosseini F, Sharifnia S, Rezaee R, Nazari R, Molukzadeh S. Cerebrovascular risk factors in type 2 diabetic patients at short term.J Ilam $U$ Med Sci. 2011;19(3):41-50.

16. Andersen KK, Andersen ZJ, Olsen TS. Age- and gender-specific prevalence of cardiovascular risk factors in 40,102 patients with first-ever ischemic stroke: a Nationwide Danish Study. Stroke. 2010;41(12):276874. doi: 10.1161/STROKEAHA.110.595785. [PubMed: 20966413].

17. Tanimura C, Matsumoto H, Tokushima Y, Yoshimura J, Tanishima S, Hagino H. Self-care agency, lifestyle, and physical condition predict future frailty in community-dwelling older people. Nurs Health Sci. 2018;20(1):31-8. doi: 10.1111/nhs.12376. [PubMed: 29115022].

18. Paganini-Hill A. Lifestyle Practices and Cardiovascular Disease Mortality in the Elderly: The Leisure World Cohort Study. Cardiol Res Pract. 2011;2011:1-7. doi: 10.4061/2011/983764.

19. Babak A, Davari S, Aghdak P, Pirhaji O. [Assessment of Healthy Lifestyle among Elderly in Isfahan, Iran]. J Isfahan Med Sch. 2011;29(149). Persian.

20. Fallah Mehrabadi E, Pakgohar M, Asadi S, Haghani H. [Lifestyle of Elderly People With Osteoporosis and Its Related Factors]. Salmand. 2017;12(2):132-45. Persian. doi: 10.21859/sija-1202132.

21. Abdi J, Eftekhar H, Mahmoodi M, Shojayzadeh D, Sadeghi R, Saber M. Effect of the Intervention Based on New Communication Technologies and the Social-Cognitive Theory on the Weight Control of the Employees with Overweight and Obesity. J Res Health Sci. 2015;15(4):25661. [PubMed: 26728913].

22. Larsson SC, Akesson A, Wolk A. Healthy diet and lifestyle and risk of stroke in a prospective cohort of women. Neurology. 2014;83(19):1699704. doi: 10.1212/WNL.0000000000000954. [PubMed: 25298305]. [PubMed Central: PMC4239835].

23. Mohammadian H, Mousavi SGA. [Lifestyle of university students in Kashan, Iran and factors affecting it].J Sch P Health Institute P Health Res. 2015;12(4):53-63. Persian.

24. Amirzadeh Iranagh J, Motalebi SA, Mohammadi F. A Theoretically Based Behavioral Nutrition Intervention for Elderly Women: A Cluster Randomized Controlled Trial. Int J Gerontol. 2017.

25. Motalebi SA, Cheong LS, Iranagh JA, Mohammadi F. Effect of low-cost resistance training on lower-limb strength and balance in institutionalized seniors. Exp Aging Res. 2018;44(1):48-61. doi: 10.1080/0361073X.2017.1398810. [PubMed: 29336735].

26. Motaghi M, Davari Dolatabadi E. Lifestyle of elderly people of Isfahan's villages in Iran in 2014. Int J Hum Cultural Stud (IJHCS). 2016:790803.

27. Momeni K, Rafiee Z. [Correlation of Social Support and Religious Orientation With Life Satisfaction in the Elderly]. Salmand. 2018;13(1):5061. Persain. doi: 10.21859/sija.13.1.50.

28. Eshaghi R, Farajzadegan Z, Babak A. Healthy lifestyle assessment questionnaire in elderly. Payesh. 2009;9(1):91-9.

29. Lori S. [A Survey on the Level of the Elderly Lifestyle in Shiraz].JHealthBased Res. Persian.

30. Heshmati H, Asnashari R, Khajavi S, Charkazi A, Babak A, Hosseini SG. [Life style of elderly in Kashmar, Iran 2012]. J Res Dev Nurs Midwifery. 2014;11(1):39-50. Persian.

31. Najimi A, Moazemi Goudarzi A. Healthy lifestyle of the elderly: Acrosssectional study. J Health Sys Res. 2012;8(4):581-7.

32. Mahmudi G, Niazazari K, Sanati T. Evaluation of life style in the elderly. (J Health Breeze) Family Health. 2013.

33. Mirghafourvand M, Mohammad-Alizadeh Charandabi S, Aliasghari F. [Assessment of health promoting lifestyle status and its sociodemographic predictors in women with polycystic ovarian syndrome]. Hayat. 2017;22(4):394-407. Persain.

34. Rezaeipandari H, Morowatisharifabad MA. Assessment of Psychosocial Determinants (Self-Efficacy and Social Support) of Lifestyle in The Elderly in Yazd City, Iran, 2015. Qom U Med Sci J. 2016;10(9):51-60. 